\title{
Photodimerization of Thymine Derivatives in Single Crystal
}

\author{
Norimitsu Tohnai, Yoshiaki Inaki ${ }^{\dagger}$ Mikiji Miyata, \\ Nobuyoshi Yasui*, Eiko Mochizuki*, and Yasushi Kai* \\ Department of Materials \& Life Science, \\ *Department of Materials Chemistry, \\ Osaka University, Yamadaoka 2-1, Suita, Osaka 565, Japan \\ †inaki@chem.eng.osaka-u.ac.jp
}

\begin{abstract}
Thymine derivatives having long alkyl chain showed a very high photoreactivity in a single crystal and gave only one isomer of photodimer while the photodimerization in solution gave the four isomers. The crystal structure determined by X-ray diffraction method suggested the formation of the trans-syn or cis-anti photodimer. The photodimer obtained, however, was identified as the trans-anti isomer from the NMR spectrum. It was concluded that the trans-anti photodimer was formed by disrotatory motion of the thymine in the crystal. Keywords: thymine, photodimerization, stereo isomer, crystal structure.
\end{abstract}

\section{INTRODUCTION}

Thymine derivatives are known to give photodimers upon irradiation to UV light near the $\lambda$ max $(280 \mathrm{~nm})$ of thymine. The photodimerization is reversible reaction and the photodimer splits to afford the original thymines very efficiently upon irradiation at a shorter wavelength $(240 \mathrm{~nm})$ (Fig. 1).<smiles>Cc1cn(P)c(=O)[nH]c1=O</smiles>

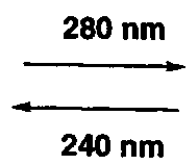

Thymine Derivative<smiles>[R]N1C(=O)NC(=O)C2(C)C1C1C(=O)NC(=O)N(P)C12</smiles>

Photodimer
Figure 1 Reversible photodimerization of the thymine derivative.

A variety of thymine derivatives were synthesized and the photoreactivity has been studied in a solid state and a polymer matrix ${ }^{1-4}$. The increase and decrease in the absorbance at $270 \mathrm{~nm}$ are repeated as shows in Fig. 2 because of the formation and cleavage of this photodimer.

In order to obtain a high sensitive photo-reversible system, it is necessary to know the reaction
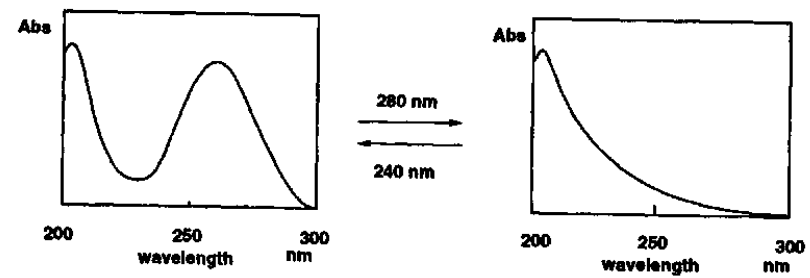

Figure 2 UV spectra of original thymine derivatives and photodimers.

mechanism of the photodimerization in the solid state. The solid of thymine derivatives obtained by spin coating, however, contained amorphous and crystalline parts ${ }^{3}$. Therefore, the photodimerization of thymine derivatives should be studied in a single crystal in order to know the reaction mechanism. This paper deals with the crystal structure analysis and photodimerization in the single crystal of the thymine compound having long alkyl chain. The photodimerization in the single crystal gave only one stereo isomer (trans-anti) of the photodimers, though the reaction in solution gave four isomers. The mechanism of the selective formation of the trans-anti isomer will be discussed considering the crystal structure of the thymine derivative. 


\section{EXPERIMENTALS}

\section{Synthesis of 1-Octyl Thymine (2)}

Thymine ( $31.5 \mathrm{~g}, 0.25 \mathrm{~mol})$ in hexamethyldisilazane (HMDS) $(250 \mathrm{ml}, 1.70 \mathrm{~mol})$ was refluxed for 24 hours at $160^{\circ} \mathrm{C}$ with $10 \mathrm{ml}$ of $\mathrm{N}, \mathrm{N}$ dimethylformamide (DMF) as a catalyst. After the reaction, DMF and HMDS were removed at $40^{\circ} \mathrm{C}$ under reduced pressure. By the distillation under reduced pressure (13 mmHg), 5-methyl-2, 4-bis(trimethylsiloxy)-pyrimidine (1) was obtained (123$125^{\circ} \mathrm{C}$, yield $98.7 \%$ ).

A mixture of 1-octyl bromide $(27.5 \mathrm{~g}, 0.142 \mathrm{~mol})$ and 5-methyl-2, 4-bis(trimethylsiloxy)-pyrimidine $(23.8 \mathrm{~g}, 0.088 \mathrm{~mol})$ was stirring for 10 days at $60^{\circ}$ C. After the reaction, methyl alcohol $(200 \mathrm{ml}), 6 \mathrm{~N}$ $\mathrm{HCl}(20 \mathrm{ml})$, and water $(100 \mathrm{ml})$ were added to the obtained oil. The mixture was heated and the precipitate was filtered. After removing the solvents under reduced pressure, the residue was extracted with water/chloroform. Chloroform was evaporated and the residue was washed with petroleum ether and recrystallized with benzene to give 2 (nearly quantitative yield). ' $\mathrm{H}$ NMR (270 MHz, $\left.\mathrm{CDCl}_{3}\right) \delta 9.04(1 \mathrm{H}$, $\mathrm{s}, \mathrm{NH}), 6.98(1 \mathrm{H}, \mathrm{s}, \mathrm{C} 6-\mathrm{H}), 3.69\left(2 \mathrm{H}, \mathrm{t}, \mathrm{N}-\mathrm{CH}_{2}\right), 1.92$ $\left(3 \mathrm{H}, \mathrm{s}, \mathrm{C} 5-\mathrm{CH}_{3}\right), 1.67\left(2 \mathrm{H}, \mathrm{m}, \mathrm{N}-\mathrm{C}-\mathrm{CH}_{2}\right), 1.25(10 \mathrm{H}$, $\left.\mathrm{m}, \mathrm{CH}_{2}\right), 0.88\left(3 \mathrm{H}, \mathrm{t}, \mathrm{C}-\mathrm{CH}_{3}\right)$.

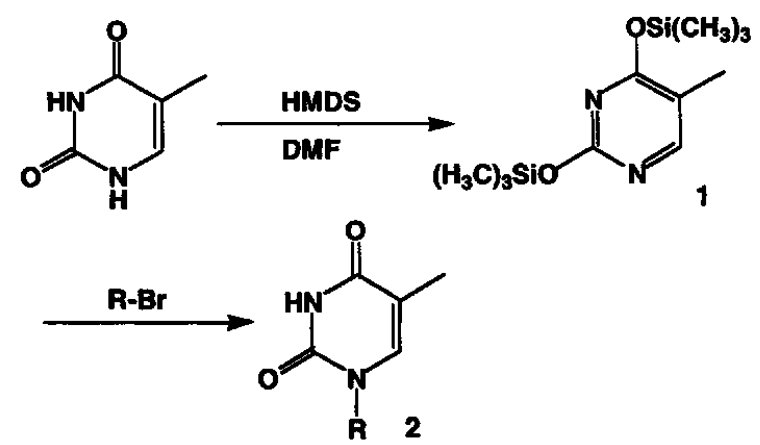

Scheme 1 Synthesis of thymine derivatives having long alkyl chain.

\section{Crystal Structure Analysis}

Crystal data for 1-octyl thymine (2) : $\mathrm{C}_{26} \mathrm{H}_{44} \mathrm{O}_{4} \mathrm{~N}_{4}$, triclinic, space group $\mathrm{P} 1, a=17.08(1), b=17.415(5)$, $c=11.756(3) \AA, \alpha=89.97(2)^{\circ}, \beta=110.02(6)^{\circ}, \gamma=$ $110.27(4)^{\circ}, V=3053(3) \AA^{3}, D$ calc $=1.04 \mathrm{~g} \mathrm{~cm}^{-3}, Z=$ $8, R=0.139$.

$\mathrm{X}$-ray diffraction intensitties of 1-octyl thymine were collected by Rigaku RAXIS-CS imaging plate two-dimensional area detector with graphitemonochromatized Mo- $K \alpha$ radiation at $15 \pm 1^{\circ} \mathrm{C}$. The 4455 unique reflections were measured $(2 \theta \max =$ $59.6^{\circ}$ ). For further calculations, $\left[\left|F_{0}\right|>3 \sigma\left|F_{0}\right|\right]$ reflections were used after Lorenz and polarization corrections. The structure was solved by direct method (SAPI 91), and refined by SHELEX 93. All nonhydrogen atoms were refined anisotropically. Hydrogen atoms attached to carbon atoms were located in the calculated positions. The positions of hydrogen atoms attached to nitrogen atoms were obtained from difference Fourier syntheses. All calculations were performed by using TEXSAN crystallographic software package of the Molecular Structure Corporation.

\section{Photodimerization}

Photodimerizations in single crystal were carried out with a super high pressure mercury lamp (SUPER CURE-203S, SAN-EI Electric). For the photodimerization in acetone solution, a high pressure mercury lamp (HALÕS P1H-500 Cl S60, EIKOSHA) was used.

The obtained four isomers were separated by recrystallization. The first crystal was obtained from methanol, and was identified as trans-anti dimer from NMR spectra (Table 1). The second crystal (trans-syn) was obtained from acetonitrile, and the third crystal (cis-syn) was obtained from ethyl acetate. The last crystal (cis-anti) was obtained from diethyl ether/petroleum ether (1/ $9, \mathrm{v} / \mathrm{v})$. The NMR data for the photodimer were identical with the data of dimethylthymine except trans-syn dimer'.

Table 1. ${ }^{1} \mathrm{H}$ NMR chemical shifts of four stereo isomeric photodimers ( $\delta$ in $\mathrm{CDCl}_{3}$ ).

\begin{tabular}{llllllll}
\hline Isomers* & $\begin{array}{llll}\mathbf{C}-\mathrm{CH}_{3} \\
6 \mathbf{6}, \mathrm{t}\end{array}$ & $\begin{array}{l}-\mathrm{CH}_{2}- \\
20 \mathrm{H}, \mathrm{m}\end{array}$ & $\begin{array}{l}5-\mathrm{CH}_{3} \\
6 \mathbf{H}, \mathrm{s}\end{array}$ & $\begin{array}{l}\mathrm{N}-\mathrm{CH}_{2} \\
2 \mathrm{H}, \mathrm{m}\end{array}$ & $\begin{array}{l}6-\mathrm{H} \\
2 \mathrm{H}, \mathrm{s}\end{array}$ & $\begin{array}{l}\mathrm{N}-\mathrm{CH}_{2} \\
2 \mathrm{H}, \mathrm{m}\end{array}$ & $\begin{array}{l}\mathrm{N}-\mathrm{H} \\
2 \mathrm{H}, \mathrm{s}\end{array}$ \\
\hline trans-anti & $\mathbf{0 . 8 7}$ & 1.27 & 1.43 & 2.84 & 3.78 & 3.90 & 7.82 \\
trans-syn & $\mathbf{0 . 8 7}$ & 1.27 & 1.44 & 2.87 & 3.52 & 3.87 & 7.92 \\
cis-syn & $\mathbf{0 . 8 7}$ & 1.26 & 1.50 & 2.53 & 3.79 & 4.01 & 8.63 \\
cis-anti & $\mathbf{0 . 8 7}$ & 1.26 & 1.59 & 2.99 & 3.34 & 3.92 & 8.26 \\
\hline
\end{tabular}

*Four stereo isomers are shown in Fig. 7. 


\section{RESULTS}

\section{Crystal Structure}

Figure 3 shows (a) the molecular packing in a unit cell and (b) the planar structure of the crystal of 1-octyl thymine. The unit cell contains eight molecules. The crystal structure is consisted of the hydrophilic layers of thymine bases and the hydrophobic layers of long alkyl groups (Fig. 4 ). The distance between the planes formed by thymine bases was almost $3.3 \AA$. By considering the van der Waals radius, the thymine bases contact approximately each other. Moreover, the long alkyl chain groups aggregate and form the hydrophobic layer.

The thymine bases are coplanar (Fig. 3b), and are associated by the hydrogen bonds on the plane (Fig.

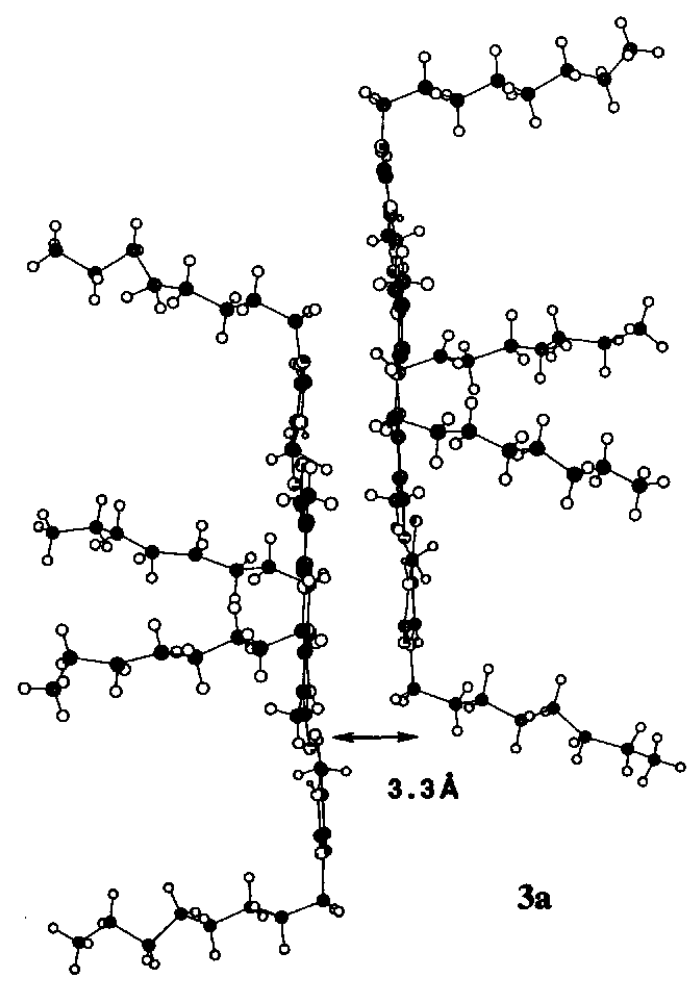

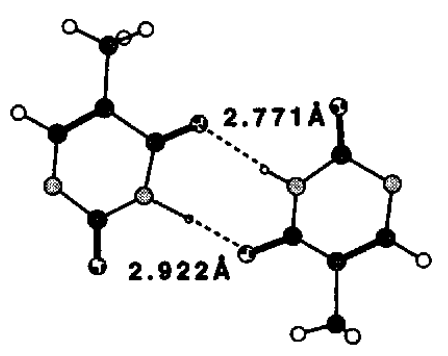

$\mathbf{3 b}$

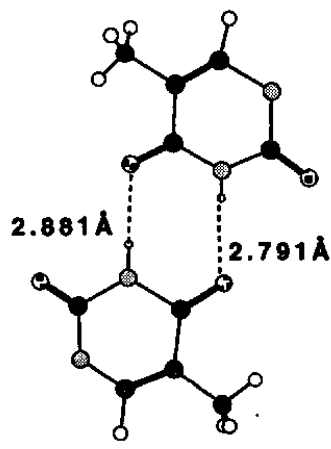

Figure 3 Crystal structure of 1-octyl thymine recrystallized from ethyl acetate.

(a) Molecular packing in the unit cell,

(b) Planar structure and hydrogen bondings.

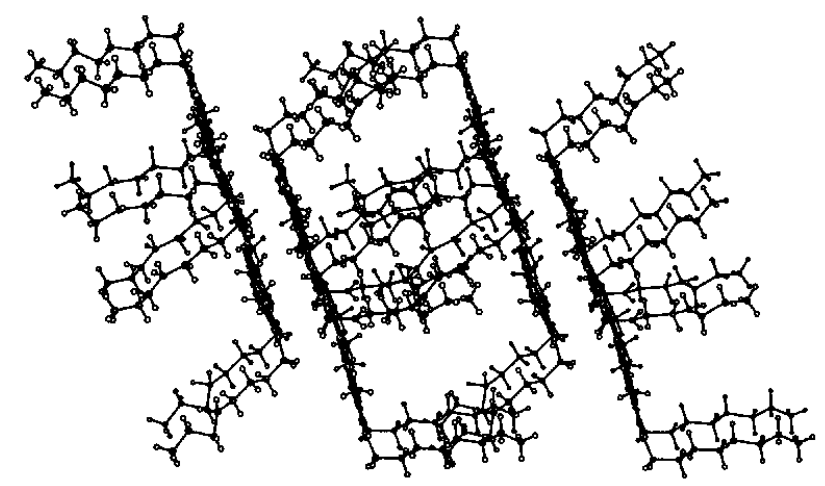

Figure 4 Lamella structure of thymine derivative having long alkyl chain.

5). The thymine molecule faces another thymine in the opposite plane with head to head (Fig. 6 ). As shown in Figure 6, two thymine bases on the opposite plane were in the position of trans-syn. Therefore, the distance and the orientation of two thymine bases in this crystal suggest that the trans-syn photodimer should be obtained dominantly by photodimerization.

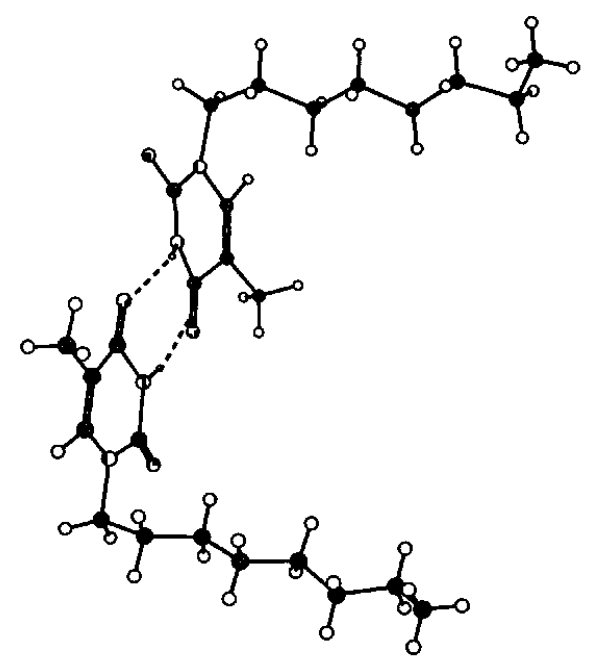

Figure 5 Two thymine derivatives associated with hydrogen bondings.

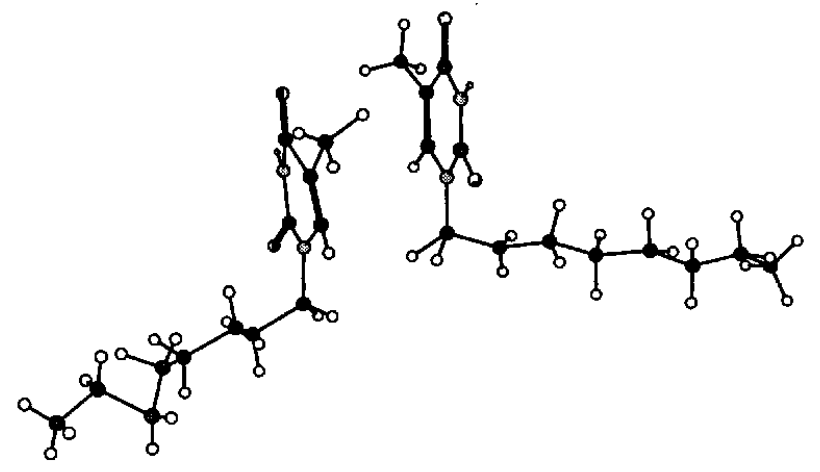

Figure 6 Two opposite thymine derivatives in the position of trans-syn. 


\section{Photodimerization}

UV light was irradiated to the plate crystal which was obtained by recrystallization from ethyl acetate. The process of the photoreaction was confirmed from UV, IR and NMR spectra. The conversion was calculated by the NMR spectra in $\mathrm{CDCl}_{3}$ from the peaks of 6-H of photodimers and original thymine. After UV irradiation of the plate crystal for 3 hours, the photodimerization proceeded in yield of $98.3 \%$.

There are four stereoisomeric dimers possible by linkage of two thymines to form a cyclobutane ring across their 5, 6 double bonds: cis-syn, cis-anti, transsyn, and trans-anti (Fig. 7). The cis-syn photodimer is known to be formed by UV irradiation on DNA.

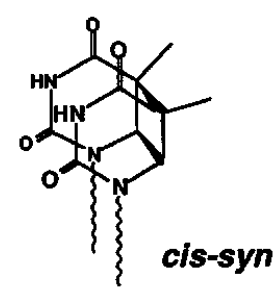<smiles>CN1C(=O)NC(=O)C2C1C1(C)C(=O)NC(=O)N2C1C1CCCCC1</smiles>

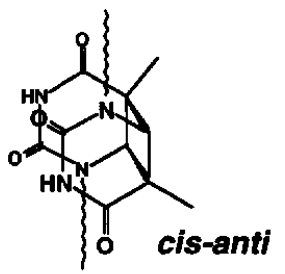

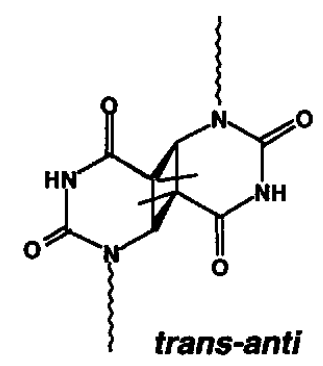

Figure 7 Four photodimer structures of 1-alkyl thymine derivatives.

The photodimerization of 1-octyl thymine hardly occurred in solution, where triplet state was the precursor $^{6-9}$. However, the photodimerization gave all isomers in acetone which was the triplet sensitizer. The isomers were isolated by recrystallization and identified from NMR (Tab. 1). In the solution reaction, the trans-anti dimer was a main product and the other isomers were generated with an almost equal ratio (Tab. 2).

Only one isomer was obtained from the UV irradiation of the plate crystal from ethyl acetate. The dimerization was fast because the reaction proceeded

Table 2. Relative photodimer distributions

\begin{tabular}{lcl}
\hline & Solution & Crystal \\
\hline cis-syn & 0.5 & - \\
\hline cis-anti & 0.6 & 0.07 \\
\hline trans-syn & 0.4 & 0.05 \\
\hline trans-anti & 1 & 1 \\
\hline
\end{tabular}

through the singlet state ${ }^{10-12}$. The isomer was identified as trans-anti isomer from chemical shifts of 5$\mathrm{CH}$ and 6-H of the NMR spectra. This isomer is different from the trans-syn isomer predicted from the crystal structure.

\section{DISCUSSION}

Crystal structure showing the nearest neighbor thymine bases in the crystal are picked out from Fig. 4 to give Fig. 8. The distance between thymines in the plane is short, but the formation of the photodimer is difficult because the interaction of double bonds is necessary for the photodimerization.

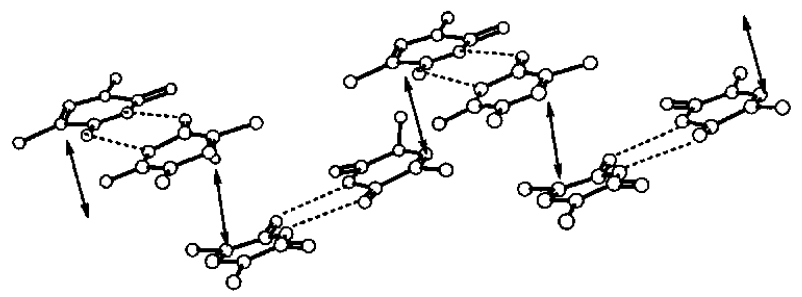

Figure 8 Hydrogen bonding network and photoreacting base pairs on the opposite plane.

Two stacked thymines in Fig. 8 are illustrated in Fig. 9. The cyclobutane photodimer can be formed by the reaction between two double bonds of $\mathrm{C} 5$ and C6. When the first bond is formed between $\mathrm{C5}$ and C5', the syn type photodimer should be formed after formation of the second bond between $\mathrm{C} 6$ and $\mathrm{C} 6$ '. On the other hand, when the first bond is formed between $\mathrm{C} 5$ and $\mathrm{C} 6$ ', the anti type photodimer should be formed after formation of the second bond between $\mathrm{C} 6$ and $\mathrm{C5}$. The crystal data suggested that the nearest carbons are $\mathrm{C} 5$ and $\mathrm{C} 6$ ' in the distance of $3.36 \AA$ as suggested in Fig. 9. Therefore, the photodimer formed by UV irradiation in the single crystal should be the anti type isomer.

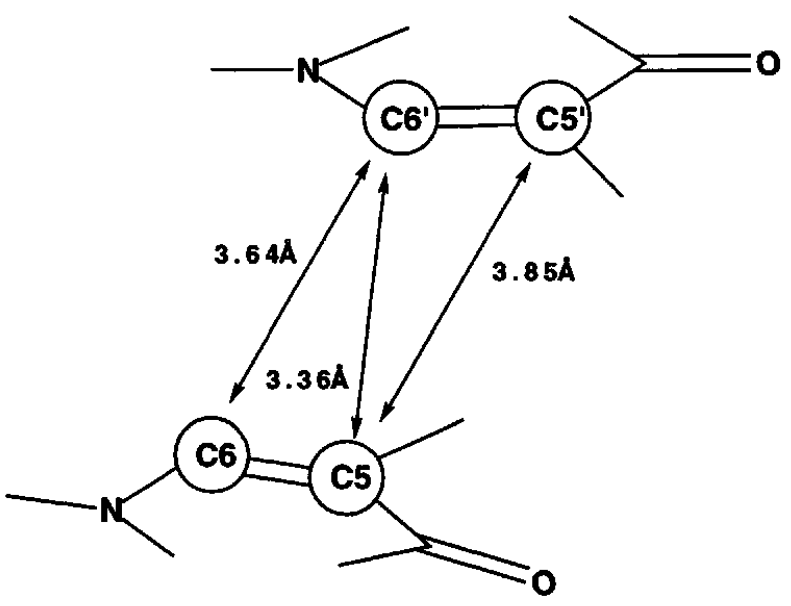

Figure 9 Photoreacting sites of the nearest neighbor thymine bases in crystal. 
If the photodimerization occurs face to face between the stacked thymines, the photodimer should be the cis-anti isomer. However, the photodimer obtained was the trans-anti isomer, suggesting another mechanism of photodimerization. The photocyclization of a diene compound is known to occur by disrotatory motion, although the thermal cyclization occurs by conrotatory motion. If the photodimerization of thymine bases in crystal occurred by disrotatory motion, the product should be the trans-anti isomer. This mechanism of disrotation may be possible because the motion of the molecule is fixed in the crystal by the interaction of the long alkyl chain.

The probable mechanism of the photodimerization is illustrated in Fig. 10. When the thymine bases in crystal rotate disrotatory (path a), the first bond is formed between $\mathrm{C} 5$ and $\mathrm{C}^{\prime}$ ' in cis conformation. After rotation of the thymine on the plane, the second bond is formed between $\mathrm{C} 6$ and $\mathrm{C}^{2}$ ' giving the trans-anti photodimer. By the conrotatory motion of thymine bases (path b), however, the cis-anti photodimer should be formed through the trans type intermediate.

Though the trans-anti isomer is the main product of the photodimerization in crystal, small amounts of another isomer (cis-anti and trans-syn) are formed (Tab. 2). These minor isomers may be formed from

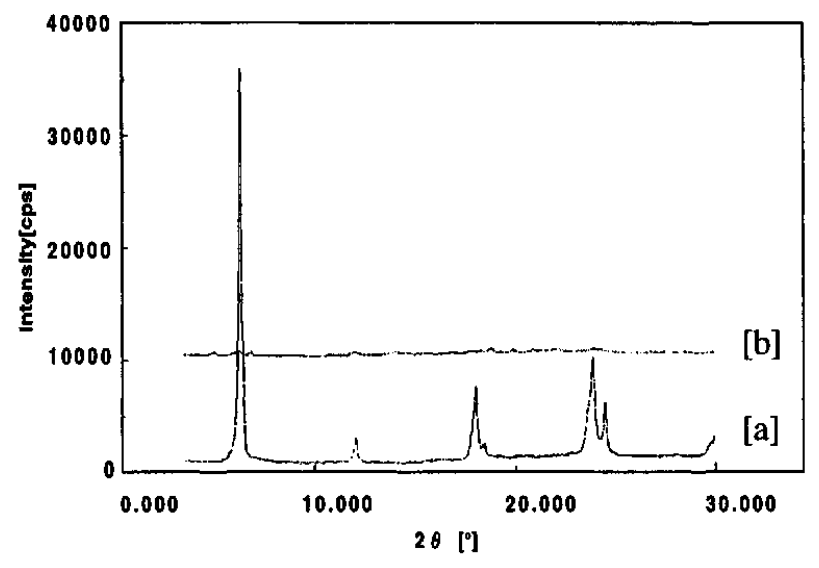

Figure 11 Powder X-ray diffraction patterns of thymine derivatives having long alkyl chain recrystallized from ethyl acetate with differ ent photoreaction conversion.

[a] conversion $=0 \%$, [b] conversion $=98.7 \%$

collapsed crystals. The powder X-ray diffraction suggested that the sharp peak observed at the beginning of the reaction became a broad peak at the end of reaction (Fig. 11). The formation of the trans-anti photodimer may accompany collapse of the crystal structure. The transparent crystal was observed to become cloudy as the progress of the reaction. It was concluded that the minor isomers obtained were formed from the collapsed crystals at the end of reaction.

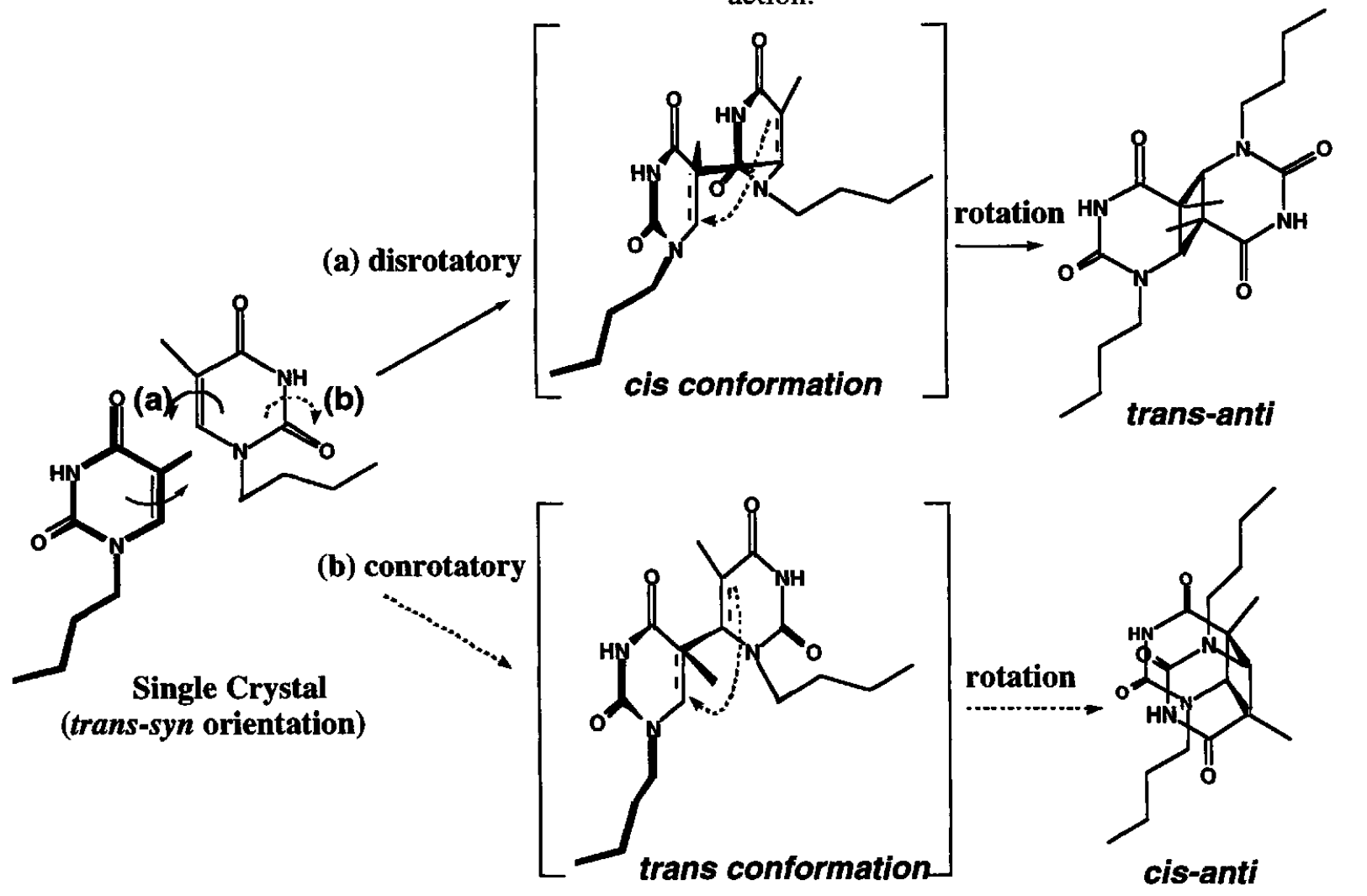

Figure 10 Probable mechanism of the photodimerization in the single crystal. 


\section{CONCLUSION}

The plate single crystal of 1-octyl thymine recrystallized from ethyl acetate showed very high photoreactivity and gave only one isomer of the photodimer. The crystal structure suggested the formation of the trans-syn or cis-anti photodimer. The photodimer obtained, however, was identified as the trans-anti isomer from the NMR spectrum. It was concluded the trans-anti photodimer was formed by disrotatory motion of the thymine in the crystal. The mechanism for the intermolecular photodimerization of thymines in the crystal was found to be the same as that for the intramolecular photocycloaddition of dienes because the thymine derivative was fixed with the alkyl group in the crystal.

\section{References}

1. Y. Inaki, Y. Wang, M. Kubo, and K. Takemoto, "Chemistry of Functional Dyes", Z. Yoshida and Y. Shirota, Eds, Mita, Osaka, p.365 (1993).
2. N. Tohnai, T. Sugiki, E. Mochizuki, T. Wada, and Y. Inaki, J. Photopolym. Sci. Technol., 7, (1994) 91. 3. T. Sugiki, N. Tohnai, E. Mochizuki, T. Wada, Y. Inaki, Bul. Chem. Soc. Jap., 69, (1996) 1777.

4. N. Tohnai, M. Miyata, Y. Inaki, J. Photopolym. Sci. Technol., 9, (1996) 63.

5. R. Kleopfer and H. Morrison, J. Am. Chem. Soc., 94, 255 (1972).

6. G. J. Fisher, and H. E. Johns, Photochem. \& Photobiol., 11, (1970) 429.

7. J. Eisinger, and A. A. Lamola, Mol. Photochem., 1, (1969) 209.

8. D. Wienblum, Photochem. Photobiol., 12, (1970) 509.

9. B. H. Jennings, S. C. Pastra, and J. L. Wellington, Photochem. \& Photobiol., 11, (1970) 215.

10. S. Y. Wang, Nature (London), 200, (1960) 879.

11. D. Weinblum, and H. E. Johns, Biochim. Biophys. Acta., 114, (1966) 450.

12. R. Lisewski, and K. L. Wierzchowski, Photochem. \& Photobiol., 11, (1970) 327. 